\title{
Porous Phosphate-Based Glass Microspheres Show Biocompatibility, Tissue Infiltration, and Osteogenic Onset in an Ovine Bone Defect Model
}

\author{
Jane S. McLaren ${ }^{\dagger, \perp}$ Laura Macri-Pellizzeri, ${ }^{\ddagger}, \perp$ Kazi M. Zakir Hossain, ${ }^{\S}$ Uresha Patel, ${ }^{\S}$ David M. Grant, ${ }^{\S}$ \\ Brigitte E. Scammell, ${ }^{, \dagger}$ Ifty Ahmed, ${ }^{*}$, and Virginie Sottile ${ }^{*}, \|_{\odot}$ \\ ${ }^{\dagger}$ Academic Orthopaedics, Trauma and Sports Medicine, Division of Rheumatology, Orthopaedics and Dermatology, School of \\ Medicine, "Wolfson STEM Centre, Division of Cancer \& Stem Cells, School of Medicine, ${ }^{\$}$ Advanced Materials Research Group, \\ Faculty of Engineering, and "Arthritis Research UK Pain Centre, University of Nottingham, Nottingham NG7 2RD, U.K.
}

Supporting Information

ABSTRACT: Phosphate-based glasses (PBGs) are bioactive and fully degradable materials with tailorable degradation rates. PBGs can be produced as porous microspheres through a single-step process, using changes in their formulation and geometry to produce varying pore sizes and interconnectivity for use in a range of applications, including biomedical use. Calcium phosphate PBGs have recently been proposed as orthobiologics, based on their in vitro cytocompatibility and ion release profile. In this study, porous microspheres made of two PBG formulations either containing $\mathrm{TiO}_{2}(\mathrm{P} 40 \mathrm{Ti})$ or without (P40) were implanted in vivo in a large animal model of bone defect. The biocompatibility and osteogenic potential of these porous materials were assessed 13 weeks postimplantation in sheep and compared to empty defects and autologous bone grafts used as negative and positive controls. Histological analysis showed marked differences between the two formulations, as lower trabeculae-like interconnection and higher fatty bone marrow content were observed in the faster degrading P40-implanted defects, while the slower degrading P40Ti material promoted dense interconnected tissue. Autologous bone marrow concentrate (BMC) was also incorporated within the P40 and P40Ti microspheres in some defects; however, no significant differences were observed in comparison to microspheres implanted alone. Both formulations induced the formation of a collagen-enriched matrix, from 20 to $40 \%$ for P40 and P40Ti2.5 groups, suggesting commitment toward the bone lineage. With the faster degrading P40 formulation, mineralization of the tissue matrix was observed both with and without BMC. Some lymphocyte-like cells and foreign body multinucleated giant cells were observed with P40Ti2.5, suggesting that this more durable formulation might be linked to an inflammatory response. In conclusion, these first in vivo results indicate that PBG microspheres could be useful candidates for bone repair and regenerative medicine strategies and highlight the role of material degradation in the process of tissue formation and maturation.

KEYWORDS: phosphate-based glasses, porous microspheres, tissue engineering, in vivo bone defect, bone regeneration

\section{INTRODUCTION}

Bone grafts, either autologous or allogenic, are currently the gold-standard strategy for the repair of bone defects due to either their intrinsic osteoconductive, osteoinductive, and/or osteointegration capacity. However, the use of autologous bone grafts $(A B G)$ presents many limitations, including donor site morbidity, limited access to large quantities, and immunocompatibility and tolerability issues related to allogenic grafts. ${ }^{1,2}$ The increasing clinical demand for an alternative bone regeneration substitute has considerably boosted the development of new materials, of either synthetic or natural origin, preferably with osteoinductive and biocompatible properties. Hydroxyapatite and $\beta$-tricalcium phosphate are already used in the clinic, showing osteoconductive properties; however, their slow degradation rate has been highlighted as a limitation to regeneration. ${ }^{3}$ Bioactive glasses have been developed as biomaterials, including silicabased glasses known as Bioglass. While silica-based glasses are slow degrading materials, phosphate-based glasses (PBGs) have significant potential as they are fully resorbable and display tailorable degradation properties (ranging from days, months to years), including formulation and geometry. ${ }^{4}$ The basic constituents of PBGs include $\mathrm{P}_{2} \mathrm{O}_{5}, \mathrm{Na}_{2} \mathrm{O}$, and $\mathrm{CaO}$ mimicking the mineral part of the bone in composition, while doping with different oxides such as $\mathrm{AgO}, \mathrm{CuO}, \mathrm{ZnO}, \mathrm{MgO}$, and $\mathrm{SrO}$ has been reported to produce specialized glasses for

Received: March 14, 2019

Accepted: April 4, 2019

Published: April 16, 2019 
Table 1. PBG Formulations Presented as mol \% for the Two Formulations Used

\begin{tabular}{lcccc} 
& \multicolumn{4}{c}{ compositional information } \\
\cline { 2 - 5 } glass code & $\mathrm{P}_{2} \mathrm{O}_{5}(\mathrm{~mol} \%)$ & $\mathrm{CaO}(\mathrm{mol} \%)$ & $\mathrm{Na}_{2} \mathrm{O}(\mathrm{mol} \mathrm{\% )}$ & $\mathrm{MgO}(\mathrm{mol} \%)$ \\
P40 & 40 & 16 & 20 & 24 \\
P40Ti2.5 & 40 & 16 & 17.5 & 24 \\
\hline
\end{tabular}

varying therapeutic applications, including the stimulation of angiogenesis, ${ }^{5}$ new bone formation, ${ }^{6}$ and antimicrobial ${ }^{7}$ and antiresorptive activities. ${ }^{8,9}$ The material degradation rate is a critical factor to provide the support required for optimal new tissue formation. An ideal bone repair material would degrade at a similar rate as that required for the formation of new bone, mimicking the creeping substitution process happening physiologically during bone remodeling. ${ }^{10}$ The addition of $\mathrm{TiO}_{2}$ has been widely used to stabilize the glass structure against rapid degradation, resulting in decreased glass solubility ultimately influencing the biological properties of the materials. ${ }^{1-13}$ Phosphate-based glasses have also been manufactured in various geometrical forms, including fibers, disks, irregular particles, and microspheres, which can also influence their degradation rates depending on the surface area. ${ }^{14}$ Material geometry can also influence the final biomedical application and selection of the optimal in vivo implantation route. Where a predefined macrostructure is not required, a regular spherical shape offers advantages in particle flow properties enabling injection via a minimally invasive delivery route in vivo. ${ }^{15}$ More importantly, manufacturing PBG microspheres with a highly porous conformation has recently been described, providing a three-dimensional bone biomimetic environment capable of supporting cell colonization and encouraging tissue infiltration. ${ }^{14}$

The hypothesis here was that by using biomimetic porous microspheres with different degradation rates it could be possible to modulate the bone tissue regeneration response. The present study investigated the potential of two porous calcium phosphate microsphere formulations, with and without $\mathrm{TiO}_{2}$ (P40Ti and $\mathrm{P} 40$, respectively), implanted in an ovine bone defect model. The PBG microspheres were also combined with autologous bone marrow concentrate (BMC) to test whether this would potentiate the biological response of the materials. The addition of autologous BMC has been considered as a possible regenerative strategy for different applications, including bone healing, due to the expected enrichment in different types of progenitor cells with regenerative potential, ${ }^{16,17}$ including mesenchymal precursors known to have osteogenic potential and exert a strong paracrine activity with regenerative effect. ${ }^{18}$ The evaluation of biocompatibility and tissue growth parameters in vivo suggests that these materials could provide a promising resource for bone repair strategies.

\section{MATERIALS AND METHODS}

The reagents used were purchased from Thermo Fisher Scientific (U.K.) unless otherwise stated.

Porous Microsphere Manufacturing and Characterization. Porous calcium phosphate glass microspheres $\left(40 \mathrm{P}_{2} \mathrm{O}_{5} \cdot 16 \mathrm{CaO}\right.$. $\left.24 \mathrm{MgO} \cdot(20-x) \mathrm{NaO} \cdot x \mathrm{TiO}_{2}(x=0,2.5 \mathrm{~mol} \%)\right)$ were manufactured using the following precursors $\mathrm{NaH}_{2} \mathrm{PO}_{4}, \mathrm{CaHPO}_{4}, \mathrm{MgHPO}_{4}, \mathrm{P}_{2} \mathrm{O}_{5}$, and $\mathrm{TiO}_{2}$ (Sigma-Aldrich, U.K.) according to the manufacturing process previously described in ref 14 (see Table 1 for glass formulations). Briefly, glass precursors were weighed and dried in a $100 \mathrm{~mL}$ volume $\mathrm{Pt} / 5 \%$ Au crucible at $350{ }^{\circ} \mathrm{C}$ for $30 \mathrm{~min}$ in an air furnace and then melted and held at $1150{ }^{\circ} \mathrm{C}$ for $90 \mathrm{~min}$ and at 1280
${ }^{\circ} \mathrm{C}$ for $120 \mathrm{~min}$ in case of Ti-containing glass. The molten glasses were then poured onto a steel plate and allowed to cool down to room temperature. Afterward, cooled glasses were ground into microparticles using a Retsch PM100 milling machine and sieved into varying sizes ranging from 63 to $125 \mu \mathrm{m}$. Porous glass microspheres were then produced by mixing the glass powder with a calcium-based porogen and processing the materials via flame spheroidization utilizing a thermal spray gun (Metallisarion, U.K.) using an oxyacetylene ratio of 3:3. Post manufacture, the porous microspheres were washed by gently stirring in $5 \mathrm{M}$ acetic acid for $2 \mathrm{~min}$, followed by deionized water for $5 \mathrm{~min}$, and then dried in an oven at $50{ }^{\circ} \mathrm{C}$ for 24 h. $^{14}$

Microsphere porosity was evaluated as described previously by Hossain et al. ${ }^{14}$ using mercury intrusion porosimetry (Micromeritics Autopore IV 9500), while the relative density was measured using a helium gas pycnometer (Micromeritics AccuPyc 1330) in approximately $1 \mathrm{~g}$ of microspheres of both formulations. For the measurement of tapped density, approximately $1 \mathrm{~g}$ of microspheres was transferred into a $10 \mathrm{~mm}$ graduated cylinder and tapped until the sample volume became constant using a Quantachrome Autotap machine. The tapped density was then calculated by dividing the mass with the final volume obtained. At least 10 measurements were conducted to derive an average density value. ${ }^{19}$ The microsphere degradation study was performed as previously described ${ }^{14}$ to calculate the percentage of mass loss at days 1, 3, 7, 14, 21, and 28 .

Preparation of Bone Marrow Concentrate and in Vivo Bone Defect Model. Twenty skeletally mature English mule ewes (age 24 years, weight $58.5-81 \mathrm{~kg}$ ) were randomly assigned to six treatment groups (Table 2). All procedures were carried out in accordance with

Table 2. Treatment Groups Included in the Study ${ }^{a}$

\begin{tabular}{llc}
\multicolumn{1}{c}{ animal group } & \multicolumn{1}{c}{ treatment received } & number of implants $(n)$ \\
P40 & P40 & 6 \\
P40 + BMC & P40 + BMC & 6 \\
P40Ti2.5 & P40Ti2.5 & 6 \\
P40Ti2.5 + BMC & P40Ti2.5 + BMC & 6 \\
ABG & autologous bone graft & 8 \\
empty & empty defect & 8
\end{tabular}

${ }^{a} \mathrm{BMC}=$ bone marrow concentrate; $\mathrm{ABG}=$ autologous bone graft.

UK Home Office Regulations and protocols and were approved by the University of Nottingham Animal Welfare and Ethical Review Body (AWERB). Autologous bone marrow aspiration was carried out under general anesthesia at the beginning of the surgical procedure as described $^{20}$ and returned within the defect, as indicated during the same anesthetic event. Anesthetized animals were placed in lateral recumbency and bone marrow aspirate was harvested from the sternum using 8 gauge $100 \mathrm{~mm}$ Jamshidi needles (UK Medical Ltd., Sheffield, U.K.) to collect aspirates in heparinized tubes. Three insertions of the Jamshidi needle were performed collecting a maximum total of $20 \mathrm{~mL}$ of bone marrow. ${ }^{20}$ Bone marrow concentrate $(\mathrm{BMC})$ samples were obtained by centrifugation of the aspirates for $30 \mathrm{~min}$ at $200 \mathrm{~g}$; pellets were then incubated with red blood cell lysis buffer (Sigma-Aldrich) for $5 \mathrm{~min}$ at room temperature to reduce the erythrocyte contaminants. Samples were washed with phosphate-buffered saline and centrifugated at $200 \mathrm{~g}$ for $5 \mathrm{~min}$. The resulting pellets were finally resuspended in cell culture medium ( $\alpha$ MEM supplemented with $10 \%$ of fetal calf serum and L-glutamine) in a final volume of $800 \mu \mathrm{L}$. 
A
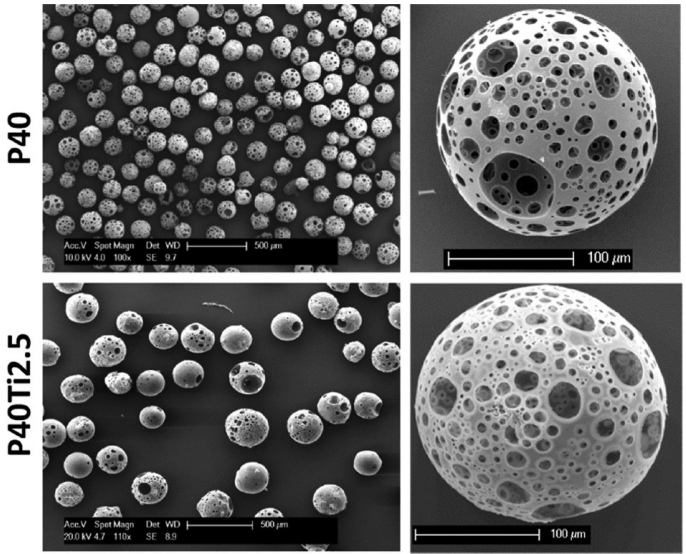

B

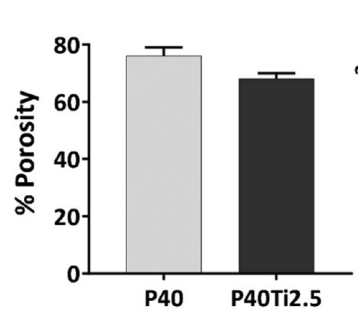

Microsphere formulations c

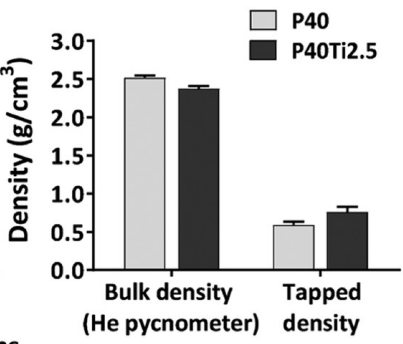

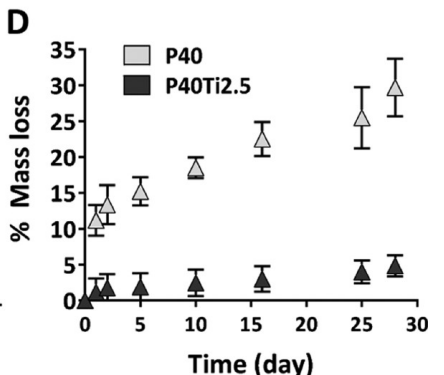

Figure 1. Properties of the PBG microspheres implanted. (A) Representative SEM images of P40 and P40Ti2.5 microspheres. (B-D) Evaluation of porosity (B), density (C), and percentage of mass loss (D) for both formulations.

A cylindrical bone defect of $8 \mathrm{~mm}$ width $\times 15 \mathrm{~mm}$ depth was drilled into the cancellous bone of medial femoral condyles of both the left and right hind legs (Suppl. Figure 1B). ${ }^{20,21}$ The drills used to create the defects were cooled using sterile saline to ensure no heat damage to the bone. Animals received P40 or P40Ti2.5 microspheres, with or without $\mathrm{BMC}$, according to following group pairing: $\mathrm{P} 40 /$ P40Ti2.5 + BMC; P40Ti2.5/P40 + BMC $(n=6)$. For material sterilization, microspheres were washed in $\mathrm{EtOH} 70 \%$, dried overnight in a vacuum oven at $50{ }^{\circ} \mathrm{C}$, and finally irradiated through Co-60 $\gamma$-irradiation (25-35 kGy radiation dose) (kindly provided by Ceramisys Ltd., U.K.). For implantation, microspheres were gently mixed with $800 \mu \mathrm{L}$ of DMEM (with or without BMC) added dropwise to produce a paste that was delivered to fill the defect site using a modified $2 \mathrm{~mL}$ syringe (Suppl. Figure 1A,B). After implantation, the filled defect was tightly covered with any available periosteum sown over the top and the release of the muscle lying over the top of the femoral condyle covering the defect, further secured by the closure of the soft tissue before suturing the skin. Defects left empty or filled with autologous bone graft $(A B G)$ were used as negative and positive controls, respectively. Bone cores harvested from the empty defects were broken into small pieces using a bone rongeur and then implanted in the $A B G$ group defects. Animals received analgesia and antibiotics as required and were closely monitored postoperatively for any clinical signs of pain. Throughout the study, body weight and body condition scores were recorded for all sheep. Body condition scores highlighted the health and amount of fat on an animal, with a score of 1 indicating an emaciated animal and a score of 5 indicating an obese animal, with a score of 3 classed as healthy. Animals were sacrificed after 13 weeks by an intravenous overdose of pentobarbital. The femoral condyles were collected and fixed in $10 \%$ neutral buffered formalin for a minimum of 7 days before further processing.

Microcomputed Tomography Analysis. A high-resolution micro-X-ray-computed tomography system (micro-CT, Skyscan 1174) was used to determine bone ingrowth within the defects in the femoral condyles. Fixed samples were scanned at a voltage of 50 $\mathrm{kV}$, a current of $800 \mu \mathrm{A}$, and a voxel resolution of $32 \mu \mathrm{m}$. PBG microsphere samples were scanned at a voxel resolution of $9 \mu \mathrm{m}$. A $0.50 \mu \mathrm{m}$ aluminum filter was also applied. A cylinder slightly larger than the defect ( $9 \mathrm{~mm}$ width $\times 15 \mathrm{~mm}$ height) was used to calculate total bone fill to ensure that any variation in the defect size was included, while an internal cylinder ( $6 \mathrm{~mm}$ width $\times 8 \mathrm{~mm}$ height $)$ was applied to measure core values and exclude callous tissue (Suppl. Figure $3 \mathrm{~A}$ ). A threshold of $255 / 70$ (threshold ${ }_{70}$ ) was selected to segment fully mineralized bone and $255 / 50$ (threshold ${ }_{50}$ ) was selected to segment both mature and immature bones from surrounding tissue. The immature bone values were obtained by the following equation

$$
\text { immature bone }=\text { threshold }_{50}-\text { threshold }_{70}
$$

Transmission images were reconstructed using Skyscan supplied software (NRecon) with the resulting two-dimensional image representing a single $32 \mu \mathrm{m}$ slice $(1 / 256)$.

Tissue Processing and Histological Analysis. Twenty-two bone samples from all treatment groups (five samples for groups $\mathrm{P} 40$ and P40 + BMC, four samples for groups P40Ti2.5 and P40Ti2.5 + BMC, two samples for empty and ABG) were dehydrated in progressively higher concentrations of ethanol solutions followed by xylene. Samples were then permeated at $4{ }^{\circ} \mathrm{C}$, resin-embedded at 11 ${ }^{\circ} \mathrm{C}$ (Technovit 9100, Kulzer), and sectioned to produce $6 \mu \mathrm{m}$-thick tissue sections using a Leica RM2255 sawing microtome. Sets of three sections were cut $150 \mu \mathrm{m}$ apart and then processed for the following staining: hematoxylin-eosin, Goldner's trichrome, and Von Kossa staining (Atlantic Bone Screen, France). Briefly, for hematoxylineosin staining, sections were immersed in hematoxylin solution and washed and stained with $10 \%$ eosin solution. Sample dehydration with ethanol and xylene solutions was performed before mounting with resinous mounting solution. For Goldner's trichrome staining, samples were stained with hematoxylin solution followed by incubation in picric acid solution. After washing, samples were stained with Fuchsin Ponceau and washed with $1 \%$ phosphomolybdic acid solution. A light green solution was used to stain the samples before dehydration in ethanol and xylene solutions and then resin mounting solution was used. For Von Kossa staining, sections were 

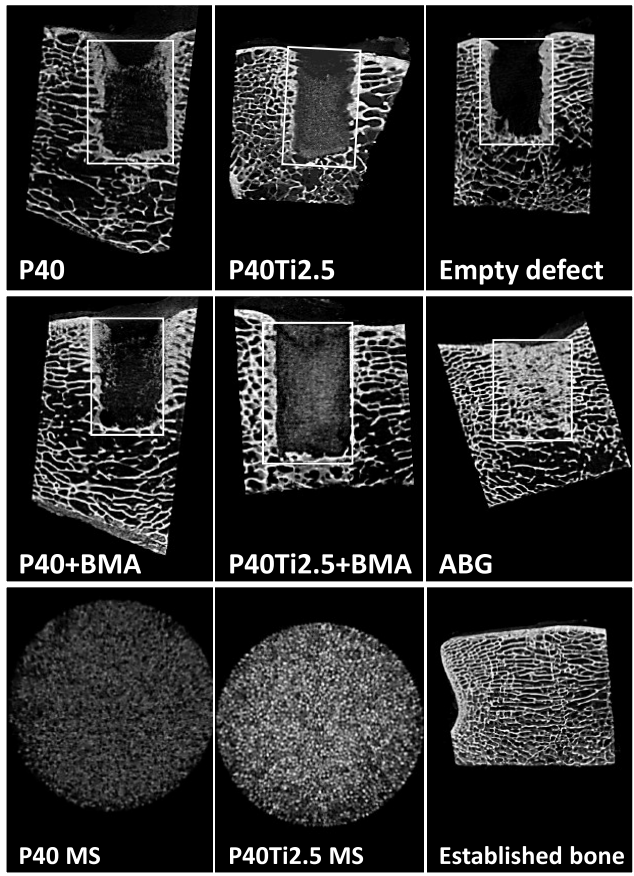

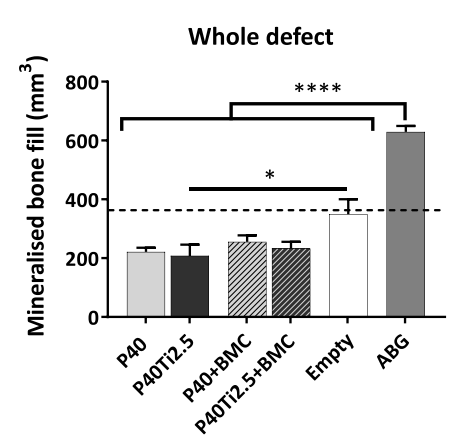

D

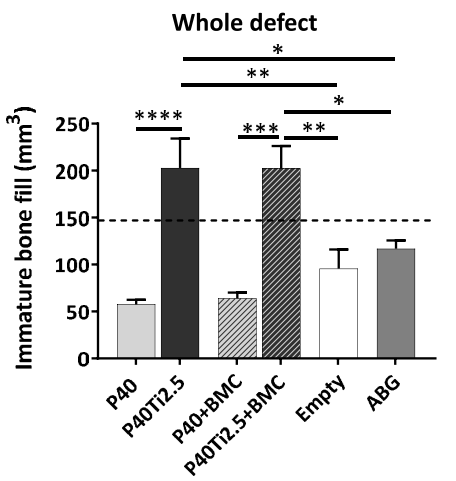

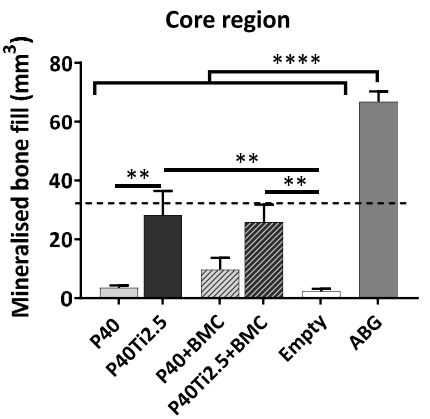

$\mathbf{E}$

Core region

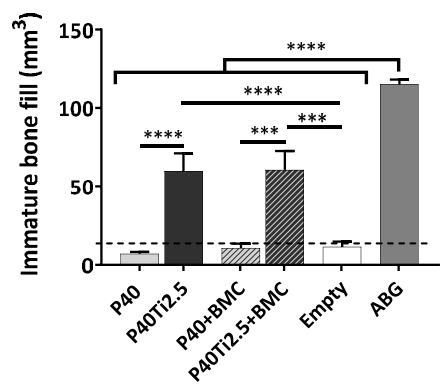

Figure 2. Micro-CT analysis. (A) Representative micro-CT cross sections of the animal groups treated with porous PBG formulations combined or not with bone marrow concentrate (BMC), negative empty control, and positive ABG control. Images of established healthy bone and ex vivo microsphere samples of both formulations were included as reference. White frame defined the defect region. (B-E) Quantification of new mineralized (B, D) and immature bones (C, E) within the defect of the six groups of treatment. Both parameters were measured either in a $9 \mathrm{~mm} \times$ $15 \mathrm{~mm}$ region of interest (occupying the whole defect) or in a core region of $6 \mathrm{~mm} \times 8 \mathrm{~mm}$. Graphs show mm ${ }^{3}$ of new tissue. ABG: autologous bone graft. Dotted lines in the graphs indicate the signal of established healthy bone. ${ }^{*} p<0.05 ; * * p<0.01 ; * * * p<0.001$; ****p<0.0001.

stained using a $1 \%$ silver nitrate solution and exposed to UV light. Excess staining was removed using a $5 \%$ sodium thiosulfate solution before counterstaining with Fuchsin Ponceau staining. Samples were dehydrated with ethanol and xylene solutions and mounted using resin mounting solution.

Quantitative image analysis was performed on Goldner's trichrome and Von Kossa stained sections using ImageJ software. Images were processed through the Feulgen Light Green plugin or the RGB plugin for Goldner's trichrome and Von Kossa stained images, respectively, to separate image color channels. For Goldner's trichrome staining, images were generated in pink, green, and orange, while for Von Kossa staining, images in green, blue, and red were obtained. For signal quantification, all images were converted to grayscale and the signal threshold was established. For Goldner's trichrome staining, green images were used for the quantification of a collagen bone-like matrix, while for the osteoid tissue, pink images were used. For Von Kossa staining, blue images were used for the evaluation of mineralized tissue content. In all cases, the calculation was done over the ROI2 surface (Suppl. Figure 3B) of two replicates per animal.

Imaging. Representative images of hematoxylin-eosin stained sections were obtained using a Nanozoomer (Nanozoomer 2.0 HT, Hamamatsu, Hamamatsu, Japan) and visualized using the NanoZoomer Digital Pathology program. For the semiquantitation of fibrous plug thickness, $4 \times$ magnification images were obtained using EVOS XL-Core system. Representative images of Goldner's trichrome and Von Kossa stained sections were taken using an EclipseT2 Nikon microscope coupled with a D3300 Nikon camera.

Statistical Analysis. Micro-CT data were analyzed using a oneway analysis of variance and a post hoc Tukey's test, while for histology data analysis, the Kruskal-Wallis nonparametric test was applied, followed by Dunn's post hoc test. All statistical analyses were carried out using the statistical package GraphPad PRISM version 7.03 , and all data are shown as mean values \pm standard error of the mean (SEM).

\section{RESULTS}

Microsphere Characterization. Two formulations of porous PBG microspheres were prepared in the system $\left(40 \mathrm{P}_{2} \mathrm{O}_{5} \cdot 16 \mathrm{CaO} \cdot 24 \mathrm{MgO} \cdot(20-x) \mathrm{NaO} \cdot x \mathrm{TiO}_{2}(x=0,2.5\right.$ mol \%)) (Table 1). ${ }^{14}$ The microsphere diameters ranged between 100 and $200 \mu \mathrm{m}$, while porosity measured $76 \% \pm 4$ for P40 and $68 \% \pm 2$ for P40Ti2.5 samples (Figure 1A,B). The average pore diameters of $\mathrm{P} 40$ and P40Ti2.5 formulations were reported to be $55 \pm 2$ and $38 \pm 4 \mu \mathrm{m}$, respectively (Suppl. Figure 2). Interestingly, the pore size distribution curve of the P40 formulation showed a wide range of values spanning from nano to micron-scale, whereas the P40Ti2.5 microspheres displayed pore size mainly in the micron range. Similar values of bulk and tapped densities were obtained for both formulations (Figure 1C). The in vitro material degradation profiles analyzed over 30 days in deionized water showed mass losses of 30 and 5\% for P40 and P40Ti2.5, respectively (Figure 1D).

In Vivo Bone Implantation. All animals displayed a good postoperative recovery, without requiring additional analgesia beyond day 3 post-surgery; no infections were observed. Weight and body condition scores were recorded for each animal before the surgery and at the 13 week end point for the monitoring of animal wellness during the study. Body weights of all animals increased an average of $6 \pm 7 \mathrm{~kg}$ over the 13 week study. All animals, that either received microspheres or were control animals, had average body condition scores at sacrifice of $3.21 \pm 0.33$ and $3.44 \pm 0.62$, respectively, with a score of 3 classed as a healthy body condition score. This 


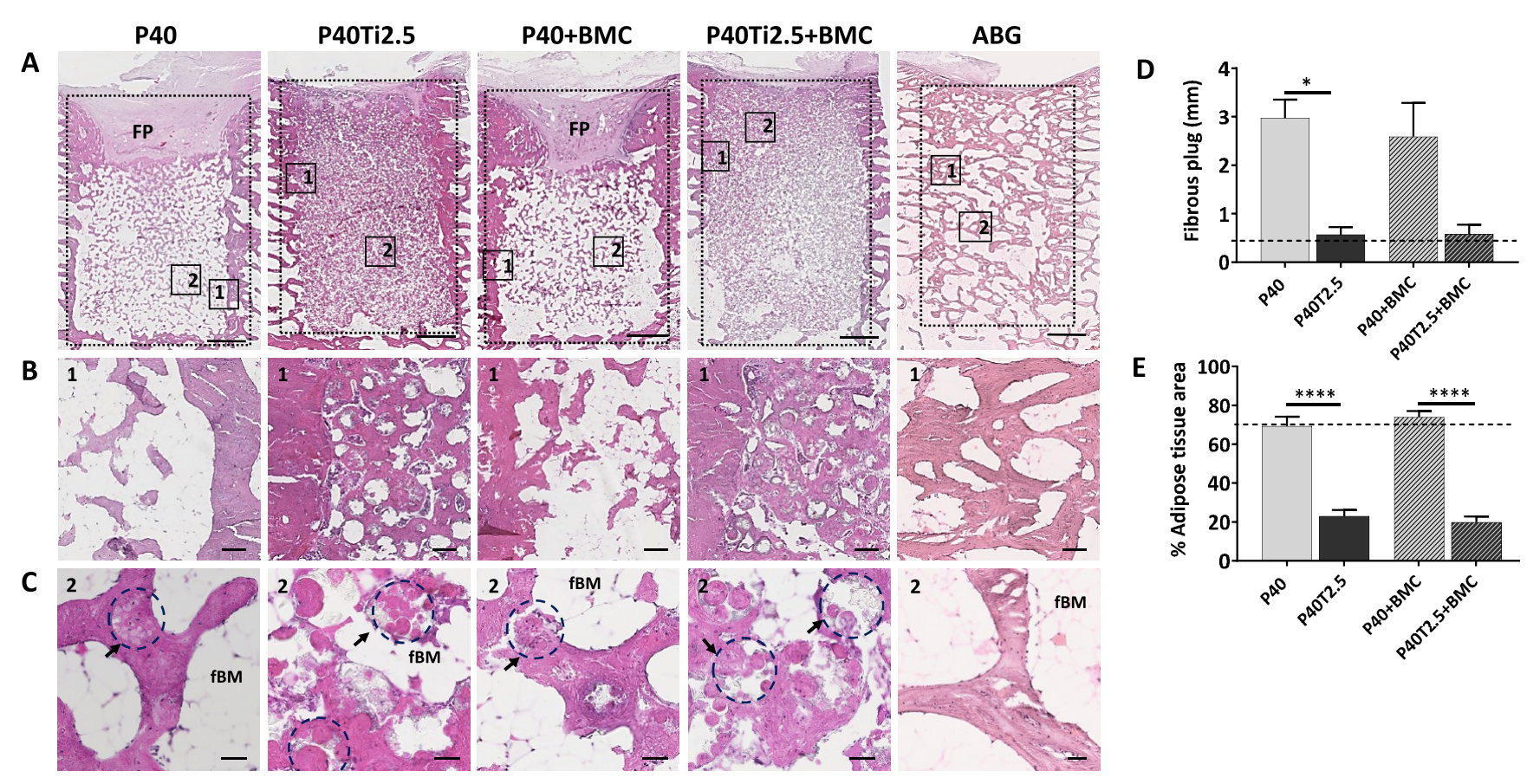

Figure 3. Hematoxylin and eosin (H\&E) staining. Representative nanozoomed images of H\&E stained tissue sections of the groups treated with the porous PBG microspheres (with and without bone marrow concentrate) and the positive ABG control. (A) Representative images at low magnification, showing the tissue organization within the entire defect area (defined by a dotted line). Insets numbered as 1 and 2 correspond to the area shown at higher magnification in (B) and (C), respectively. Scale bar: $2 \mathrm{~mm}$. (B) Zoomed-in area at the edge between the defect and endogenous bone. Scale bar: $150 \mu \mathrm{m}$. (C) Detail of the organization and tissue structure in the inner area of the defects. Black arrows indicate new tissue filling porous microspheres (dotted circles). Scale bar: $50 \mu \mathrm{m}$. (D) Semiquantitation of the fibrous plug at the entrance of the defect. (E) Semiquantitation of the \% area within the defect covered by fatty bone marrow tissue. Dotted lines in (D) and (E) indicate the values obtained for the ABG positive control group. FP: fibrous plug; ABG: established bone graft; fBM: fatty bone marrow. ${ }^{*} p<0.05$; $* * * *<0.0001$.

suggested that no issues were encountered with the safety of the procedure and the materials tested.

Evaluation of Bone Formation through Microcomputed Tomography. The formation of immature and mineralized bones was quantified within all defects through micro-CT analysis at the 13 week time point. Representative micro-CT images for all animal groups as well as for both microsphere formulations scanned ex vivo setting the same parameters used for the bone samples (255/50 and 255/70) are shown in Figure 2A. A thick mineralized border was detected at the edge of the defect for all of the groups that received materials as well as the empty group. Interestingly, a strong signal was detected particularly for the P40Ti2.5 formulation (Figure 2A). Evaluation of mineralized bone over the whole defect showed significantly higher values for the ABG positive control group in comparison to those for all other groups $(p<0.0001)$, including the empty negative control, confirming the effectiveness of the model (Figure 2B). Despite the lack of mineralization in the central area, high micro-CT values were obtained for the empty negative control, which might be due to the pronounced mineralized border observed (Figure 2A,B). Therefore, the second smaller (6 mm $\times 8 \mathrm{~mm}$ ) core region of interest was considered for all samples to evaluate the mineralization in the center of the defect and exclude the defect border (Suppl. Figure 3A). When the analysis was performed over this region, both groups treated with P40Ti2.5 showed significantly higher values in comparison to the empty negative control $(p<0.01)$, and a significant difference was also observed between the P40Ti2.5 and P40 formulations $(p<0.01)$, while the ABG control presented significantly higher values in comparison to all other treatment groups $(p<0.0001)$ (Figure $2 \mathrm{C}$ ).

The evaluation of immature bone content was also performed over the same regions of interest (Suppl. Figure $3 \mathrm{~A})$. In this case, significantly higher values were observed for both P40Ti2.5 groups (P40Ti2.5 and P40Ti2.5 + BMC) in comparison to the P40 counterparts (P40Ti2.5 vs P40: $p<$ 0.0001; P40Ti2.5 + BMC vs P40 + BMC: $p<0.001)$ and the negative empty control (P40Ti2.5/P40Ti2.5 + BMC vs empty: $p<0.01$ ) in both the whole defect and core region (Figure 2D,E). Also, while the ABG positive control showed a significantly lower amount of immature bone in comparison to the P40Ti2.5 groups in the whole defect region, an opposite trend was observed for the core region, where the signal was significantly higher in comparison to all other groups ( $p<$ 0.0001 ).

Histological Tissue Assessment. All samples were stained with hematoxylin and eosin for the evaluation of tissue formation, structure, and organization (Figure 3). The edges between the defect and the surrounding tissue appeared to be linear without any signs of osteolysis (Figure 3A). A marked fibrous plug was present at the top of the defect in both groups treated with P40 microspheres (P40 and P40 + BMC), while this was significantly thinner in both P40Ti2.5 and P40Ti2.5 + $\mathrm{BMC}$ groups and in the ABG positive control (Figure 3A,D). While the formation of new connective tissue, stained in pale pink in $\mathrm{H} \& \mathrm{E}$ staining, was detected in all PBG groups, this was observed to be denser, more developed, and interconnected in both P40Ti2.5-treated groups (Figure 3A,C). In these groups, a certain degree of physical and structural continuity could be seen between the new tissue and the surrounding endogenous 

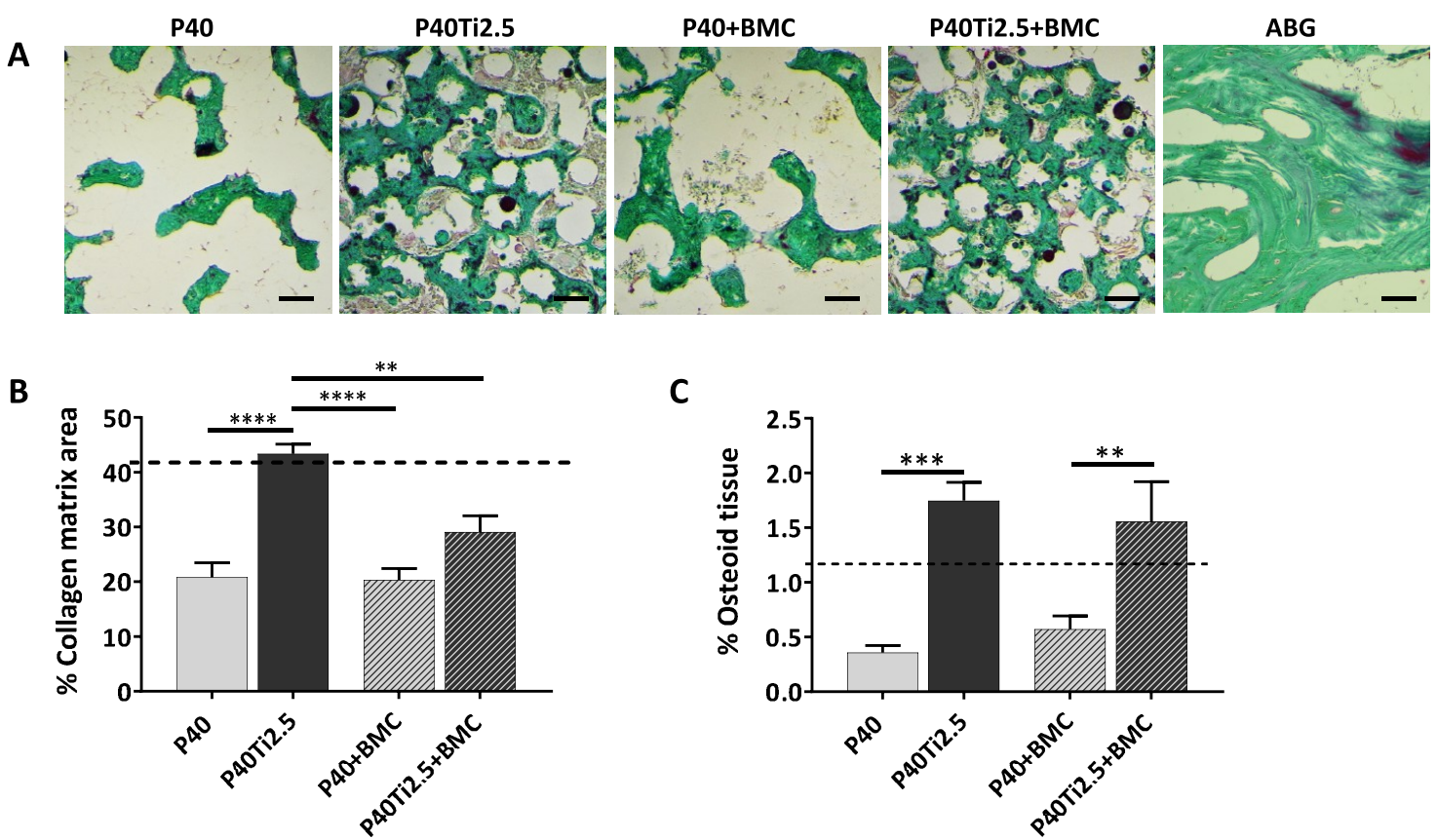

Figure 4. Goldner's trichrome staining and semiquantification of collagen and osteoid tissue content. (A) Representative images of tissue sections stained with Goldner's Trichrome to evaluate the presence of bone-like collagen (green) and osteoid tissue (red). (B) Semiquantitation of collagen and osteoid tissue content $(\mathrm{C})$ in the four groups. Dotted lines indicate the values obtained in the positive ABG group. Graphs show the \% over the total surface of soft tissue within the defect (ROI2). ABG: autologous bone graft. Scale bar: $125 \mu \mathrm{m} * * p<0.01, * * * p<0.001, * * * * p<0.0001$.

tissue, which could be attributed to the presence of the porous microspheres providing support to the connective tissue and maintaining contact with the surrounding bone tissue (Figure 3B). Conversely, in P40-treated animals, the connective tissue resembled organized trabeculae-like structures in addition to mineralized bone spicules, which were sparse and isolated without significant interaction with the surrounding bone. In the P40 groups, abundant fatty marrow tissue was observed in the space between the trabeculae-like growth within the defect, resulting in significantly higher amounts in comparison to the other groups $(p<0.0001)$ (Figure $3 \mathrm{C}, \mathrm{E})$. It is worth noting that residual microspheres were visible within the defects treated with P40Ti2.5, and new connective tissue was seen to have developed around the microspheres, invading the pore structure (Figure 3C, black arrows), while in P40-treated defects, the material was less visible and seemed to have resorbed.

Evaluation of Bone-like Collagen and Immature Osteoid Tissue Content. The amount of bone matrix collagen and osteoid tissue within the defect was assessed through Goldner's trichrome staining (Figure 4A). Semiquantitation showed that collagen (in green) occupied between 20 and $40 \%$ of the entire defect across all PBG groups, and significantly higher values were observed for the group treated with P40Ti2.5 in comparison to both P40treated groups $(p<0.0001)$ as well as to P40Ti2.5 + BMC $(p$ $<0.01$ ) (Figure 4B). Moreover, low-level staining for osteoid tissue was observed (visible in red) across all formulations (Figure 4C). Despite the low values detected, significantly higher amounts of osteoid tissue were present in both groups treated with P40Ti2.5 in comparison to the P40-treated groups (P40Ti2.5 vs P40: $p<0.001 ; \mathrm{P} 40 \mathrm{Ti} 2.5+\mathrm{BMC}$ vs $\mathrm{P} 40+$ BMC: $p<0.01)$.

Evaluation of Inflammatory Response. The possible induction of an inflammatory response evoked by the implanted material was also evaluated in Goldner's trichrome stained sections (Figure 5). Small lymphoid-like inflammatory

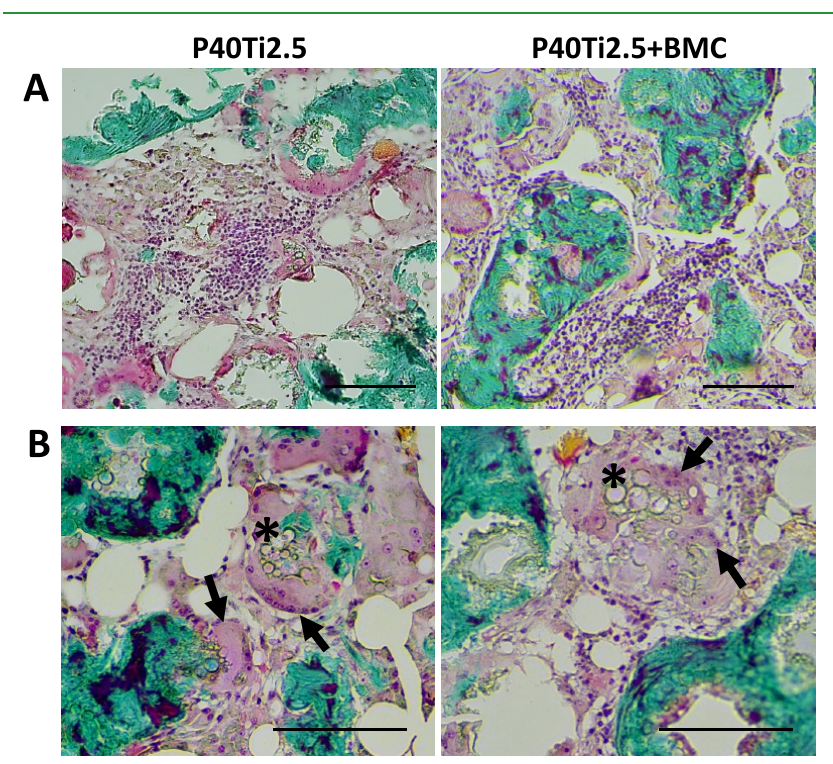

Figure 5. Images of inflammatory cells within the defect of P40Tiand P40Ti2.5-treated groups. (A) Representative images of inflammatory lymphoid cells within the defect of the groups treated with both P40Ti2.5 formulations. (B) Multinucleated cells (arrows) were also observed in proximity of nondegraded microsphere residue (asterisk). Scale bar: $125 \mu \mathrm{m}$.

cells were observed in two out of four animals in the P40Ti2.5 group and three out of four animals in the P40Ti2.5 + BMC group, while they were not observed in animals treated with the P40 formulations or in the ABG positive control (Figure 5A). Within P40Ti2.5-treated groups, numerous multinucleated giant cells (MNGCs) were found in close proximity 


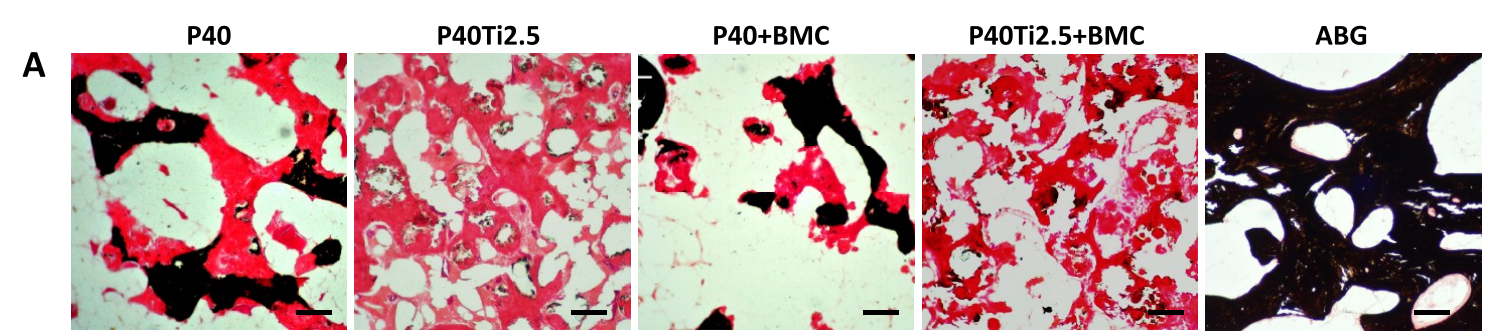

B

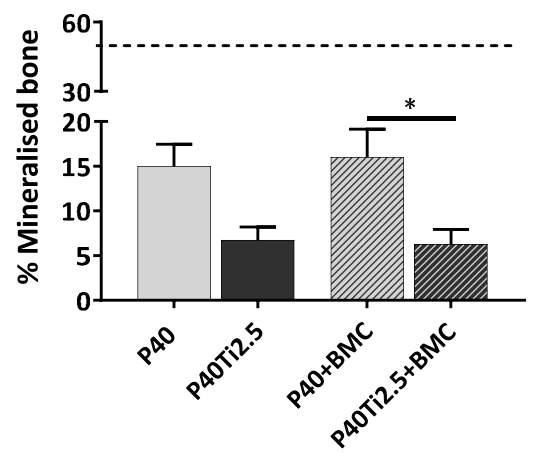

Figure 6. Von Kossa staining and quantification. (A) Representative images of tissue sections stained with Von Kossa for the detection of mineralized tissue (black) in the four groups. (B) Semiquantitation of mineralized tissue indicated as \% over the total surface of the entire defect (ROI2). Dotted line indicates the value obtained in the positive ABG control. Scale bar: $125 \mu \mathrm{m}$. ABG: autologous bone graft. ${ }^{*} p<0.05$.

to nondegraded microspheres (Figure 5B, arrows and stars, respectively).

Evaluation of Mineralized Bone Fill. Von Kossa staining was performed to evaluate the content of mineralized bone (stained in black) (Figure 6A). Both groups that were treated with $\mathrm{P} 40$ microspheres, with or without BMC, presented large and defined black-stained areas within the trabeculae-like structures observed in the defect. Conversely, a different staining pattern was observed for the P40Ti2.5 and P40Ti2.5 + $\mathrm{BMC}$ groups, where the positive black signal was limited to the residual nondegraded microspheres (Figure 6A and Suppl. Figure 4). Semiquantitation of the mineralized tissue fraction over the total surface of the defect confirmed these observations with higher values for both groups treated with P40 (Figure 6B), although only the difference between P40 + $\mathrm{BMC}$ and $\mathrm{P} 40 \mathrm{Ti} 2.5+\mathrm{BMC}$ groups reached statistical significance $(p<0.05)$.

\section{DISCUSSION}

This study describes the biocompatibility and osteogenic response to two quaternary formulations of phosphate-based glass (PBG) from the system $40 \mathrm{P}_{2} \mathrm{O}_{5} \cdot 16 \mathrm{CaO} \cdot 24 \mathrm{MgO} \cdot(20-$ $x) \mathrm{NaO} \cdot x \mathrm{TiO}_{2}(x=0,2.5 \mathrm{~mol} \%)$ (Table 1$)$ manufactured as novel porous microspheres utilizing a single-step manufacturing process. As shown recently, the porous P40 microspheres supported in vitro cell growth, adhesion, and colonization of the pores using an immortalized line of hMSCs. ${ }^{14}$ In line with this observation, newly formed connective tissue was observed in this study within the pores of microspheres after 13 weeks. This new tissue was observed to grow within a threedimensional construct. This is consistent with studies where porous materials have been considered superior for tissue regeneration in comparison to their nonporous counterpart due to enhanced tissue ingrowth and infiltration and vascularization. $^{22,23}$ Several studies have attempted to define the influence of material pore size on the outcome of new bone formation in vivo, with contrasting results. For instance, when comparing ceramic scaffolds with different pore sizes between 50 and $500 \mu \mathrm{m}$, larger values were associated with more bone formation. ${ }^{24-26}$ However, an opposite trend has been reported in a recent study by Diao and colleagues, with $100 \mu \mathrm{m}$ pore scaffolds performing better than 250 and $400 \mu \mathrm{m}$ pore devices. $^{27}$ In this study, the Ti-free P40 formulation presented slightly larger pores than the P40Ti2.5, which could be due to the higher glass density for the latter. As previously shown, the range of pore size obtained $(30-50 \mu \mathrm{m})$ is suitable to promote cell migration and colonization. ${ }^{14}$

The addition of small quantities of $\mathrm{TiO}_{2}$ to the glass formulation is a known strategy for the control of PBG degradation rates. ${ }^{9,12}$ In our study, the decreased degradation rate (only 5\% degradation was observed after 30 days for P40Ti as opposed to $30 \%$ for P40) was associated with delayed resorption of the P40Ti2.5 microspheres, which were still visible after 13 weeks, while the P40 microspheres appeared fully resorbed. These observations are in agreement with previous reports that the timing of material degradation can significantly influence the outcome of the therapeutic approach. $^{28,29}$

The histological analysis revealed marked differences between defects treated with $\mathrm{Ti}$-free and $\mathrm{Ti}$-containing porous PBG microspheres. The first visible difference induced by the two formulations was the density of the connective tissue within the defects, which was visibly higher in the P40Ti2.5treated groups. This tissue detected in P40Ti2.5-treated groups also appeared to have a different structure in comparison to that in P40-treated ones. Despite the trabeculae-like architecture visible in P40-treated groups, these presented low or no interconnection with each other or with the surrounding bone tissue. In the P40Ti groups, the connective tissue was widely distributed around and inside the residual nondegraded microspheres with no trabecular-like organization, which suggested that the long-term presence of nondegraded materials, beyond stimulating more tissue formation, may have delayed the tissue maturation and bone 
remodeling process. ${ }^{30}$ The formation of trabeculae is essential for the correct bone functionality. Parameters such as trabecular number, trabecular separation, and distribution have been associated with higher incidence of fractures in a cohort of female individuals affected by osteoporosis, while a specific intertrabecular angle characterizes healthy bone, highlighting the importance of the correct structural tissue organization. ${ }^{31,32}$

In addition to the different amounts of connective tissue induced by the two formulations tested in this study at 13 weeks, they also induced different levels of mineralization in the respective treatment groups, with a higher amount of mineralized tissue detected in groups treated with the P40 formulation. PBGs represent instructive materials as the ions released during the degradation process may act as determinants of biological processes, with the ability to influence stem cell differentiation and promote mineralization. In particular, the principal ions released by the materials tested in this study (calcium, magnesium, sodium, and phosphate) have been described to exert influential effects on osteoblast cells, including the induction of proliferation and mineralization. ${ }^{9,33,34}$ Studies also indicated that not only the type of ions released but also the relative amounts released from the material are critical for tissue regeneration. ${ }^{35}$ Based on these considerations, it is reasonable to consider that the different degradation rates characterizing the two formulations studied here affected tissue formation through both an instructive and a structural effect. It would now be useful to analyze the in situ effect of the material degradation on specific lineages, including osteoblasts and osteoclasts, to fully characterize the nature of the tissue response.

Results of the mineralized bone content obtained through Von Kossa staining appeared to partially contradict the results provided by the micro-CT analysis, which initially showed significantly higher values for both the P40Ti2.5 groups. However, the strong signal observed from the ex vivo scan for both microsphere formulations suggested that the micro-CT values might be overestimating the new mineralized bone because of the high intrinsic signal from the P40Ti2.5 material itself, which could be due to the $\mathrm{Ti}$ incorporated within the formulation. $^{36,37}$ It would thus be of interest to consider extending the follow-up period (6-12 months) so that the long-term effect of the formulations on defect mineralization could be untangled from the presence of the remaining material.

Another marked difference between the two formulations highlighted by the histological analysis was the presence of lymphoid-like inflammatory cells within the defects in P40Ti2.5-treated tissues. ${ }^{38}$ As part of the adaptive immunity, these cells play a role in wound healing and body response to biomaterials and foreign bodies. ${ }^{39}$ Beyond the morphological identification, a cell marker analysis for the specific lineages (e.g., Th1 and Th2 lymphocytes) present in the tissue could provide further information on the possible pro- or antiinflammatory nature of such a response, as these cells are known to produce different panels of cytokines. ${ }^{40,41}$ Moreover, cells resembling multinucleated giant cells (MNGCs) were observed in direct contact with nondegraded materials, which were more prominent in the Ti-treated groups, as expected from their slower degradation rate. These foreign body MNGCs have intrinsic phagocytic activity and could be involved in the physical elimination of the residual materials from the defect. ${ }^{42}$ Evidence from in vitro studies suggested that the physical properties of the materials, including particle size and material composition, may stimulate such a phagocytic activity. Milde and colleagues observed that larger particles exerted an inductive effect on these cells, while the addition of calcium phosphate glass containing $5 \mathrm{~mol} \%$ of $\mathrm{TiO}_{2}$ to a polymeric scaffold, which reduced its degradation rate together with high surface erosion and debris release, was reported to stimulate a significantly higher number of foreign body MNGCs than the glass-free counterpart, in line with the observations made in this study. ${ }^{43,44}$ Further analysis using specific immunological markers would help to fully characterize cell types and understand their role in the body response to the implanted materials. Barbeck and co-workers observed the expression of the integrin-beta2 receptor in foreign body MNGC phagocytic cells in the presence of a HA-based synthetic material (NanoBone) but not in osteoclast-like cells in contact with bone matrix. ${ }^{42}$ The detection of osteoclasts using TRAP staining, which was not possible here due to the sample-processing procedure, could also allow the assessment of resorption activity in the treated defects. It is important to highlight that beyond the inflammatory cellular infiltrate observed within the defects, this local reaction did not appear to affect animal behavior, body score, and weight over the study period, which indicated the general safety of the surgical approach and the biocompatibility of the materials tested.

Our results also indicate that the addition of bone marrow concentrate (BMC) did not appear to yield any significant additional beneficial effect for the parameters analyzed. This was unexpected since the use of autologous BMC is largely accepted as an advantageous cell therapy strategy for different applications, including bone healing, due to the expected enrichment in mesenchymal precursors with osteogenic potential among other progenitors present. ${ }^{16,17}$ The absence of significant benefit for BMC in the present study could be due to a suboptimal amount of stem cells introduced to the defects, or to the insufficient engraftment of the cells after delivery to the defect. A prospective clinical trial performed in 2011 showed the ability of BMC to promote complete bone healing in bone defects of $\geq 1 \mathrm{~cm} \times 1 \mathrm{~cm}$ when combined with either a hydroxyapatite scaffold or with a collagen sponge. ${ }^{45}$ In this study, the authors performed a 15 min incubation to allow the cells to adhere to the carrier material; in this way, the dispersion of the cells once implanted in vivo could be prevented, possibly increasing the retention of the implanted cells in situ and therefore their efficacy.

Beside the bioactivity and the osteointegration shown in this study, these porous microspheres are characterized by low mechanical strength making them compactable and thus particularly suitable for defect filling. These features are shared with other materials largely used in the clinic, including hydroxyapatite (HA), $\beta$-tricalcium phosphate $(\beta$-TCP), and silicate-based bioglasses. HA shows good osteoconductivity and biocompatibility; however, it is poorly resorbable $(5.4 \%$ after 6 months), affecting the quality and mechanical strength of the new tissue. ${ }^{46} \beta$-TCP is regarded as being more degradable than $\mathrm{HA}$, resembles the structure and mechanical properties of cancellous bone, and may take up to 24 months to fully resorb. ${ }^{47}$ It is mainly used in the clinic as a bone defect filler. $^{48,49}$ The silicate-based bioglasses (such as 45S5, 13-93, and S53P4) are also available on the market for bone regeneration, showing good compatibility and integration with the host tissue. ${ }^{50} \mathrm{~A}$ study comparing the regenerative ability of bioglasses $45 \mathrm{~S} 5, \mathrm{HA}$, and $\beta$-TCP in a goat radius 
bone defect showed superior regenerative results, including higher bone growth rate and osteointegration, for the $45 \mathrm{S5}$ bioglass scaffold in comparison to $\mathrm{HA}$ and $\beta$-TCP, ${ }^{3}$ highlighting the importance of ion release and material bioactivity. From these materials already used in the clinic, the porous PBG microspheres presented in this study provide significant advantage of being highly tailorable in both geometry, formulation, and hence degradation profiles. In addition, their controllable bioresorbability is a highly desired property for tissue engineering application, as it can be progressively substituted by native tissue restoring physiological functionality. It would also prevent the complications related to the long-term presence of foreign bodies in the organism. ${ }^{51}$ As such, these highly porous PBG microsphere biomaterials constitute a promising alternative to the existing materials for bone tissue engineering application.

In summary, this study utilizing a sheep knee bone defect model showed that porous phosphate-based glass microspheres are good candidates for tissue regeneration application in vivo, as they are biocompatible and promoted new bone tissue formation and osteointegration. For these properties, they indeed constitute a valid alternative to the use of bone graft for bone regeneration, although this is still considered the golden standard, showing a more powerful performance in terms of the amount and quality of new bone formed due to their natural and biomimetic features. Bone grafts are indeed rich in many of those growth factors known to be key in tissue regeneration, including BMP-2, BMP-4, FGF, PDGF, and IGF1 among others, but also to provide the optimal structural support through the ECM network to promote not only new tissue formation and maturation but also vascularization and host tissue integration resulting in very high quality outcomes. ${ }^{52,53}$ Considering these results, alternate formulations with an intermediate degradation rate tailored to more closely match the kinetics of bone regeneration and remodeling processes would represent an excellent strategy to achieve higher amounts of mineralized tissue with significantly more developed trabecular organization. A follow-up study with a longer end point beyond the 13 week period analyzed here would also be useful to allow slower degrading formulations to fully resorb and provide better support for completion of the remodeling process.

\section{CONCLUSIONS}

This study evaluated the biological potential of two PBG formulations, with or without titanium, manufactured as porous microspheres suitable for injection in minimally invasive procedures. Both materials were observed to be biocompatible, and their different degradation rates were associated with a clearly different tissue response, confirming the importance of this parameter among others in the design of new biomaterials. From the two formulations tested, the faster degrading P40 formulation produced poor trabecular-like interconnection, which may negatively affect tissue functionality. Conversely, the slow degrading P40Ti2.5 microspheres, which were still visible within the host tissue at the 13 week end point, promoted dense interconnected tissue but also delayed tissue maturation and remodeling. Therefore, the development of a formulation with an intermediate degradation profile could provide a valuable strategy to further improve the tissue regenerative response to the material and its potential for bone repair.

\section{ASSOCIATED CONTENT}

\section{Supporting Information}

The Supporting Information is available free of charge on the ACS Publications website at DOI: 10.1021/acsami.9b04603.

Microsphere implantation model (Figure S1); pore size and distribution (Figure S2); regions of interest used for semi-quantitative analysis (Figure S3); and nondegraded PBG material observed in P40Ti2.5 and P40Ti2.5 + BMC groups (Figure S4) (PDF)

\section{AUTHOR INFORMATION}

\section{Corresponding Author}

*E-mail: virginie.sottile@nottingham.ac.uk. Phone: +44 115 8231235 (V.S.).

ORCID $\odot$

Virginie Sottile: 0000-0002-6064-5738

\section{Author Contributions}

${ }^{\perp}$ J.S.M. and L.M.-P. contributed equally to the publication.

Notes

The authors declare no competing financial interest.

\section{ACKNOWLEDGMENTS}

This work was supported by the Engineering and Physical Sciences Research Council [Grant Number EPSRC EP/ K503800/1]. We are grateful for the expertise of Michael Baker and Deborah Surgay (University of Nottingham) and their invaluable support for the surgical study. Veterinary input from Diane Storer and advice from Prof Emad Rakha are also gratefully acknowledged. Histological processing of bone samples was carried out at Atlantic Bone Screen, France.

\section{REFERENCES}

(1) Roberts, T. T.; Rosenbaum, A. J. Bone Grafts, Bone Substitutes and Orthobiologics: the Bridge between Basic Science and Clinical Advancements in Fracture Healing. Organogenesis 2012, 8, 114-124.

(2) Egol, K. A.; Nauth, A.; Lee, M.; Pape, H. C.; Watson, J. T.; Borrelli, J., Jr. Bone Grafting: Sourcing, Timing, Strategies, and Alternatives. J. Orthop. Trauma 2015, 29, S10-S14.

(3) Ghosh, S. K.; Nandi, S. K.; Kundu, B.; Datta, S.; De, D. K.; Roy, S. K.; Basu, D. In Vivo Response of Porous Hydroxyapatite and Betatricalcium Phosphate Prepared by Aqueous Solution Combustion Method and Comparison with Bioglass Scaffolds. J. Biomed. Mater. Res., Part B 2008, 86B, 217-227.

(4) Abou-Neel, E. A.; Pickup, D. M.; Valappil, S. P.; Newport, R. J.; Knowles, J. C. Bioactive Functional Materials: a Perspective on Phosphate-based Glasses. J. Mater. Chem. 2009, 19, 690-701.

(5) Gérard, C.; Bordeleau, L. J.; Barralet, J.; Doillon, C. J. The Stimulation of Angiogenesis and Collagen Deposition by Copper. Biomaterials 2010, 31, 824-831.

(6) Leem, Y. H.; Lee, K. S.; Kim, J. H.; Seok, H. K.; Chang, J. S.; Lee, D. H. Magnesium Ions Facilitate Integrin Alpha 2- and Alpha 3mediated Proliferation and Enhance Alkaline Phosphatase Expression and Activity in hBMSCs. J. Tissue Eng. Regener. Med. 2016, 10, E527E536.

(7) Bellantone, M.; Williams, H. D.; Hench, L. L. Broad-spectrum Bactericidal Activity of $\mathrm{Ag}(2) \mathrm{O}$-doped Bioactive Glass. Antimicrob. Agents Chemother. 2002, 46, 1940-1945.

(8) Shahnazari, M.; Sharkey, N. A.; Fosmire, G. J.; Leach, R. M. Effects of Strontium on Bone Strength, Density, Volume, and Microarchitecture in Laying Hens. J. Bone Miner. Res. 2006, 21, 1696-1703.

(9) Hoppe, A.; Guldal, N. S.; Boccaccini, A. R. A Review of the Biological Response to Ionic Dissolution Products from Bioactive Glasses and Glass-ceramics. Biomaterials 2011, 32, 2757-2774. 
(10) Newton, C. D.; Nunamaker, D. M. Textbook of Small Animal Orthopaedics; JB Lippincott, 1985.

(11) Navarro, M.; Ginebra, M. P.; Planell, J. A. Cellular Response to Calcium Phosphate Glasses with Controlled Solubility. J. Biomed. Mater. Res. 2003, 67A, 1009-1015.

(12) Abou Neel, E. A.; Chrzanowski, W.; Knowles, J. C. Effect of Increasing Titanium Dioxide Content on Bulk and Surface Properties of Phosphate-based Glasses. Acta Biomater. 2008, 4, 523-534.

(13) Abou Neel, E. A.; Mizoguchi, T.; Ito, M.; Bitar, M.; Salih, V.; Knowles, J. C. In Vitro Bioactivity and Gene Expression by Cells Cultured on Titanium Dioxide Doped Phosphate-based Glasses. Biomaterials 2007, 28, 2967-2977.

(14) Hossain, K. M. Z.; Patel, U.; Kennedy, A. R.; Macri-Pellizzeri, L.; Sottile, V.; Grant, D. M.; Scammell, B. E.; Ahmed, I. Porous Calcium Phosphate Glass Microspheres for Orthobiologic Applications. Acta Biomater. 2018, 72, 396-406.

(15) Mitragotri, S.; Burke, P. A.; Langer, R. Overcoming the Challenges in Administering Biopharmaceuticals: Formulation and Delivery Strategies. Nat. Rev. Drug Discovery 2014, 13, 655-672.

(16) Herten, M.; Grassmann, J. P.; Sager, M.; Benga, L.; Fischer, J. C.; Jager, M.; Betsch, M.; Wild, M.; Hakimi, M.; Jungbluth, P. Bone Marrow Concentrate for Autologous Transplantation in Minipigs. Characterization and Osteogenic Potential of Mesenchymal Stem Cells. Vet. Comp. Orthop. Traumatol. 2013, 26, 34-41.

(17) Jager, M.; Jelinek, E. M.; Wess, K. M.; Scharfstadt, A.; Jacobson, M.; Kevy, S. V.; Krauspe, R. Bone Marrow Concentrate: a Novel Strategy for Bone Defect Treatment. Curr. Stem Cell Res. Ther. 2009, 4, 34-43.

(18) Knight, M. N.; Hankenson, K. D. Mesenchymal Stem Cells in Bone Regeneration. Adv. Wound Care 2013, 2, 306-316.

(19) Gupta, D.; Hossain, K. M. Z.; Ahmed, I.; Sottile, V.; Grant, D. M. Flame-Spheroidized Phosphate-Based Glass Particles with Improved Characteristics for Applications in Mesenchymal Stem Cell Culture Therapy and Tissue Engineering. ACS Appl. Mater. Interfaces 2018, 10, 25972-25982.

(20) Markides, H.; M'Laren, J. S.; Telling, N. D.; Alom, N.; AlMutheffer, E. A.; Oreffo, R. O. C.; Zannettino, A.; Scammell, B. E.; White, L. J.; El Haj, A. J. Translation of Remote Control Regenerative Technologies for Bone Repair. npj Regener. Med. 2018, 3, No. 9.

(21) McLaren, J. S.; White, L. J.; Cox, H. C.; Ashraf, W.; Rahman, C. V.; Blunn, G. W.; Goodship, A. E.; Quirk, R. A.; Shakesheff, K. M.; Bayston, R.; Scammell, B. E. A Biodegradable Antibiotic-impregnated Scaffold to Prevent Osteomyelitis in a Contaminated In Vivo Bone Defect Model. Eur. Cells Mater. 2014, 27, 332-349.

(22) Liu, X.; Rahaman, M. N.; Fu, Q. Bone Regeneration in Strong Porous Bioactive Glass (13-93) Scaffolds with an Oriented Microstructure Implanted in Rat Calvarial Defects. Acta Biomater. 2013, 9, 4889-4898.

(23) Velasco, M. A.; Narvaez-Tovar, C. A.; Garzon-Alvarado, D. A. Design, Materials and Mechanobiology of Biodegradable Scaffolds for Bone Tissue Engineering. Biomed. Res. Int. 2015, 2015, No. 729076.

(24) Karageorgiou, V.; Kaplan, D. Porosity of 3D Biomaterial Scaffolds and Osteogenesis. Biomaterials 2005, 26, 5474-5491.

(25) Chang, B. S.; Lee, C. K.; Hong, K. S.; Youn, H. J.; Ryu, H. S.; Chung, S. S.; Park, K. W. Osteoconduction at Porous Hydroxyapatite with Various Pore Configurations. Biomaterials 2000, 21, 1291-1298.

(26) Gauthier, O.; Bouler, J. M.; Aguado, E.; Pilet, P.; Daculsi, G. Macroporous Biphasic Calcium Phosphate Ceramics: Influence of Macropore Diameter and Macroporosity Percentage on Bone Ingrowth. Biomaterials 1998, 19, 133-139.

(27) Diao, J.; OuYang, J.; Deng, T.; Liu, X.; Feng, Y.; Zhao, N.; Mao, C.; Wang, Y. 3D-Plotted Beta-Tricalcium Phosphate Scaffolds with Smaller Pore Sizes Improve In Vivo Bone Regeneration and Biomechanical Properties in a Critical-Sized Calvarial Defect Rat Model. Adv. Healthcare Mater. 2018, 7, No. 1800441.

(28) Yu, X.; Tang, X.; Gohil, S. V.; Laurencin, C. T. Biomaterials for Bone Regenerative Engineering. Adv. Healthcare Mater. 2015, 4, $1268-1285$.
(29) Vishwakarma, A.; Bhise, N. S.; Evangelista, M. B.; Rouwkema, J.; Dokmeci, M. R.; Ghaemmaghami, A. M.; Vrana, N. E.; Khademhosseini, A. Engineering Immunomodulatory Biomaterials to Tune the Inflammatory Response. Trends Biotechnol. 2016, 34, $470-482$.

(30) Sanzana, E. S.; Navarro, M.; Macule, F.; Suso, S.; Planell, J. A.; Ginebra, M. P. Of the In Vivo Behavior of Calcium Phosphate Cements and Glasses as Bone Substitutes. Acta Biomater. 2008, 4, 1924-1933.

(31) Reznikov, N.; Chase, H.; Ben Zvi, Y.; Tarle, V.; Singer, M.; Brumfeld, V.; Shahar, R.; Weiner, S. Inter-trabecular Angle: A Parameter of Trabecular Bone Architecture in the Human Proximal Femur that Reveals Underlying Topological Motifs. Acta Biomater. 2016, 44, 65-72.

(32) Sornay-Rendu, E.; Boutroy, S.; Munoz, F.; Delmas, P. D. Alterations of Cortical and Trabecular Architecture are Associated with Fractures in Postmenopausal Women, Partially Independent of Decreased BMD Measured by DXA: the OFELY Study. J. Bone Miner. Res. 2007, 22, 425-433.

(33) Valerio, P.; Pereira, M. M.; Goes, A. M.; Leite, M. F. The Effect of Ionic Products from Bioactive Glass Dissolution on Osteoblast Proliferation and Collagen Production. Biomaterials 2004, 25, 29412948.

(34) Murphy, W. L.; McDevitt, T. C.; Engler, A. J. Materials as Stem Cell Regulators. Nat. Mater. 2014, 13, 547-557.

(35) Maeno, S.; Niki, Y.; Matsumoto, H.; Morioka, H.; Yatabe, T.; Funayama, A.; Toyama, Y.; Taguchi, T.; Tanaka, J. The Effect of Calcium Ion Concentration on Osteoblast Viability, Proliferation and Differentiation in Monolayer and 3D Culture. Biomaterials 2005, 26, 4847-4855.

(36) Gabet, Y.; Bab, I. A Validated Method for Titanium Implant Anchorage Analysis using MicroCT and Biomechanical Testing. Adv. Tech. Biol. Med. 2016, 4, No. 180.

(37) Li, J. Y.; Pow, E. H.; Zheng, L. W.; Ma, L.; Kwong, D. L.; Cheung, L. K. Quantitative Analysis of Titanium-induced Artifacts and Correlated Factors During Micro-CT Scanning. Clin. Oral Implants Res. 2014, 25, 506-510.

(38) Al-Nabulsi, M.; Basnet, A.; Salerno, V.; Cholankeril, M. A Case of Mantle Cell Lymphoma Presenting with Ascites. Clin. Case Rep. 2016, 4, 399-403.

(39) Spits, H.; Di Santo, J. P. The Expanding Family of Innate Lymphoid Cells: Regulators and Effectors of Immunity and Tissue Remodeling. Nat. Immunol. 2011, 12, 21-27.

(40) Abbas, A. K.; Murphy, K. M.; Sher, A. Functional Diversity of Helper T Lymphocytes. Nature 1996, 383, 787-793.

(41) Sallusto, F.; Lenig, D.; Forster, R.; Lipp, M.; Lanzavecchia, A. Two Subsets of Memory $\mathrm{T}$ Lymphocytes with Distinct Homing Potentials and Effector Functions. Nature 1999, 401, 708-712.

(42) Barbeck, M.; Booms, P.; Unger, R.; Hoffmann, V.; Sader, R.; Kirkpatrick, C. J.; Ghanaati, S. Multinucleated Giant Cells in the Implant Bed of Bone Substitutes are Foreign Body Giant Cells- New Insights into the Material-mediated Healing Process. J. Biomed. Mater. Res., Part A 2017, 105, 1105-1111.

(43) Milde, R.; Ritter, J.; Tennent, G. A.; Loesch, A.; Martinez, F. O.; Gordon, S.; Pepys, M. B.; Verschoor, A.; Helming, L. Multinucleated Giant Cells Are Specialized for ComplementMediated Phagocytosis and Large Target Destruction. Cell Rep. 2015, 13, 1937-1948.

(44) Barbeck, M.; Serra, T.; Booms, P.; Stojanovic, S.; Najman, S.; Engel, E.; Sader, R.; Kirkpatrick, C. J.; Navarro, M.; Ghanaati, S. Analysis of the In Vitro Degradation and the In Vivo Tissue Response to Bi-layered 3D-printed Scaffolds Combining PLA and Biphasic PLA/bioglass Components - Guidance of the Inflammatory Response as Basis for Osteochondral Regeneration. Bioact. Mater. 2017, 2, 208223.

(45) Jäger, M.; Herten, M.; Fochtmann, U.; Fischer, J.; Hernigou, P.; Zilkens, C.; Hendrich, C.; Krauspe, R. Bridging the Gap: Bone Marrow Aspiration Concentrate Reduces Autologous Bone Grafting in Osseous Defects. J. Orthop. Res. 2011, 29, 173-180. 
(46) Carson, J. S.; Bostrom, M. P. Synthetic Bone Scaffolds and Fracture Repair. Injury 2007, 38, S33-S37.

(47) Artzi, Z.; Weinreb, M.; Givol, N.; Rohrer, M. D.; Nemcovsky, C. E.; Prasad, H. S.; Tal, H. Biomaterial Resorption Rate and Healing Site Morphology of Inorganic Bovine Bone and Beta-tricalcium Phosphate in the Canine: a 24-month Longitudinal Histologic Study and Morphometric Analysis. Int. J. Oral Maxillofac. Implants 2004, 19, 357-368.

(48) Finkemeier, C. G. Bone-grafting and Bone-graft Substitutes. J. Bone Jt. Surg., Am. Vol. 2002, 84, 454-464.

(49) Pryor, L. S.; Gage, E.; Langevin, C. J.; Herrera, F.; Breithaupt, A. D.; Gordon, C. R.; Afifi, A. M.; Zins, J. E.; Meltzer, H.; Gosman, A.; Cohen, S. R.; Holmes, R. Review of Bone Substitutes. Craniomaxillofac. Trauma Reconstr. 2009, 2, 151-160.

(50) Välimäki, V. V.; Aro, H. T. Molecular Basis for Action of Bioactive Glasses as Bone Graft Substitute. Scand. J. Surg. 2006, 95, 95-102.

(51) Haycock, J. W. 3D Cell Culture: a Review of Current Approaches and Techniques. Methods Mol. Biol. 2011, 695, 1-15.

(52) Pape, H. C.; Evans, A.; Kobbe, P. Autologous Bone Graft: Properties and Techniques. J. Orthop. Trauma 2010, 24, S36-S40.

(53) Khan, S. N.; Cammisa, F. P., Jr.; Sandhu, H. S.; Diwan, A. D.; Girardi, F. P.; Lane, J. M. The Biology of Bone Grafting. J. Am. Acad. Orthop. Surg. 2005, 13, 77-86. 